\title{
Piezoresistivity Characterization of Synthetic Silicon Nanowires Using a MEMS Device
}

\author{
Yong Zhang, Student Member, IEEE, Xinyu Liu, Member, IEEE, Changhai Ru, Member, IEEE, \\ Yan Liang Zhang, Member, IEEE, Lixin Dong, Senior Member, IEEE, and Yu Sun, Senior Member, IEEE
}

\begin{abstract}
This paper presents a microelectromechanical systems (MEMS) device for simultaneous electrical and mechanical characterization of individual nanowires. The device consists of an electrostatic actuator and two capacitive sensors, capable of acquiring all measurement data (force and displacement) electronically without relying on electron microscopy imaging. This capability avoids the effect of electron beam (e-beam) irradiation during nanomaterial testing. The bulk-microfabricated devices perform electrical characterization at different mechanical strain levels. To integrate individual nanowires to the MEMS device for testing, a nanomanipulation procedure is developed to transfer individual nanowires from their growth substrate to the device inside a scanning electron microscope. Silicon nanowires are characterized using the MEMS device for their piezoresistive as well as mechanical properties. It is also experimentally verified that e-beam irradiation can significantly alter the characterization results and must be avoided during testing.

[2011-0031]
\end{abstract}

Index Terms-Electrical characterization, mechanical characterization, microelectromechanical systems (MEMS) tensile testing, nanomanipulation, piezoresistivity characterization, scanning electron microscope (SEM), silicon nanowires.

\section{INTRODUCTION}

C HARACTERIZATION of nanomaterials is important for understanding their properties and exploring their applications. Among a range of properties of nanomaterials, the piezoresistive effect is of interest because of its potential utilization in electromechanical sensors and strain engineering

Manuscript received February 3, 2011; revised April 1, 2011; accepted April 26, 2011. Date of publication June 9, 2011; date of current version August 3, 2011. This work was supported by Hitachi High-Technologies Canada Inc., by the Natural Sciences and Engineering Research Council of Canada under a Collaborative Research and Development Grant, by the Ontario Centers of Excellence under a Collaborative Research Grant, by the Canada Research Chairs Program, and by Canadian Microelectronics Corporation under a Financial Assistance Program for Microfabrication. Subject Editor Y. Zohar.

Y. Zhang and Y. L. Zhang are with the Advanced Micro and Nanosystems Laboratory, University of Toronto, Toronto, ON M5S 3G8, Canada (e-mail: yzhang@mie.utoronto.ca; yanliang.zhang@utoronto.ca).

$X$. Liu is with the Department of Mechanical Engineering, McGill University, Montreal, QC H3A 2K6, Canada (e-mail: xinyu.liu@mcgill.ca).

$\mathrm{C}$. Ru is with Robotics and Microsystems Center, Soochow University, Suzhou 215021, China (e-mail: changhai.ru@utoronto.ca).

L. Dong is with the Department of Electrical and Computer Engineering, Michigan State University, East Lansing, MI 48824, USA (e-mail: ldong@egr. msu.edu).

Y. Sun is with the Advanced Micro and Nanosystems Laboratory, University of Toronto, Toronto, ON M5S 3G8, Canada, and also with the Robotics and Microsystems Center, Soochow University, Suzhou 215021, China (e-mail: sun@mie.utoronto.ca).

Color versions of one or more of the figures in this paper are available online at http://ieeexplore.ieee.org.

Digital Object Identifier 10.1109/JMEMS.2011.2153825 for nanoelectronic applications. For instance, individual singlewalled carbon nanotubes were used as active transducer elements in a pressure sensor [1] and a displacement sensor [2].

To quantify the piezoresistivity of individual nanomaterials, a number of experimental techniques have been reported. The experiments in common require simultaneous mechanical loading and electrical measurement of a nanomaterial. For example, the cantilever tip of an atomic force microscope (AFM) was used to laterally push a single-walled carbon nanotube suspended over a trench between two solid electrodes [3], which results in large local deformation of the nanotube at the tube-tip contact point. To achieve more uniform tensile stretching, carbon nanotubes were suspended between a solid terrace and a suspended beam, which was pushed downward by an AFM cantilever [4]. This scheme also results in large local deformation of carbon nanotubes at the edges of the terrace and suspended beam, which undesirably contributes to the characterization results.

Uniaxial or almost uniaxial tensile strains were also produced. The four-point bending method was used to deform silicon nanowires that were epitaxially grown across a trench, producing low-level uniform strains on the order of $10^{-4}$ [5]. To produce severe strains, silicon nanowires were grown between a silicon pad and a cantilever beam, which was pushed by a probe along the longitudinal direction of the nanowire [6]. A single-walled carbon nanotube was adsorbed on top of a membrane and was electromechanically characterized through bulge testing [1]. A silicon nanowire was embedded close to the anchor of a cantilever, which was pushed down by a stylus [7]. Individual InGaAs/GaAs nanosprings were stretched by two nanomanipulators inside a scanning electron microscope (SEM) for piezoresistivity characterization [8].

In addition to the aforementioned methods, since microelectromechanical systems (MEMS) actuators are capable of delivering adequate motion resolutions and ranges for deforming nanomaterials, a number of MEMS devices were developed for mechanical characterization of nanomaterials [9]-[24]. For example, electrostatic actuators [10], [13], [20] and electrothermal actuators [13], [22] were utilized to stretch nanomaterial specimens. MEMS capacitive sensors were incorporated into some of the MEMS devices [11], [20] to measure tensile forces of the specimen. The elongation measurements of the nanomaterial were obtained via SEM imaging.

While most of existing MEMS devices are only capable of mechanical characterization of nanomaterials, two of them have electrical testing capabilities [16], [17]. However, the device reported in [16] can only characterize a pair of nanowires in conjunction, rather than individual nanowires, because the 
two electrical terminals are both on the stationary portion of the device. The device reported in [17] contains an electrothermal actuator, which causes temperature increase in the specimen and affects nanomaterial characterization results. Additionally, force sensing mechanisms of both devices [16], [17] are coupled with actuators, which can introduce large errors in comparison with the use of an independent force sensor.

This paper reports on a MEMS device and experimental techniques for the electrical and mechanical characterization of individual 1-D nanomaterials. Different from previous MEMS tensile-testing devices, this device is capable of acquiring both force and elongation data of a nanomaterial specimen electronically without relying on SEM imaging. In addition, since this MEMS device is capable of performing electrical measurements on a nanomaterial under controllable mechanical strains, the piezoresistive property of the nanomaterial is able to be characterized. The effect of electron beam (e-beam) irradiation on the characterization results is also presented. Electrical insulation on the suspended structures was created through a microfabrication process, enabling the electrical measurement of a nanomaterial. Individual silicon nanowires were integrated to the MEMS device via pick-and-place nanomanipulation inside a SEM and characterized for their mechanical and piezoresistive properties.

\section{Device Design And Analysis}

\section{A. Device Design}

As schematically illustrated in Fig. 1(a) and (b), the device is composed of two suspended shuttles, namely, the actuator shuttle (on the left) and the force sensor shuttle (on the right), with a small gap in between to be bridged by a nanomaterial specimen. The actuator shuttle includes an electrostatic actuator and a capacitive displacement sensor (lateral comb-drive configuration) that measures displacements of the actuator. The force sensor shuttle contains a capacitive force sensor (transverse-comb configuration), which measures tensile forces of the specimen as well as its own displacement. When the actuator shuttle moves leftward, the specimen is stretched and the force sensor shuttle is also pulled leftward. Thus, the amount of specimen elongation is the displacement of the actuator subtracted by that of the force sensor.

This electronic method of elongation measurement has advantages over electron microscopy (EM) imaging as used in previous MEMS-based nanomaterial testing devices. First, it offers a higher sampling rate than EM imaging (EM typically has a rate of 13 frames/s in fast scanning mode). Thus, data points during rapidly developing events (e.g., plastic deformation and failure) can possibly be captured. Second, EM imaging can be relieved from observing the entire nanomaterial and, hence, can be used to focus on a section of the nanomaterial with a higher magnification (e.g., for in situ study of deformation mechanisms). Third, EM imaging affects electromechanical characterization, owing to e-beam irradiation. We experimentally verified the e-beam irradiation effect, which will be discussed quantitatively in Section IV-D. Fourth, the specimen can be characterized outside the EM vacuum chamber, making
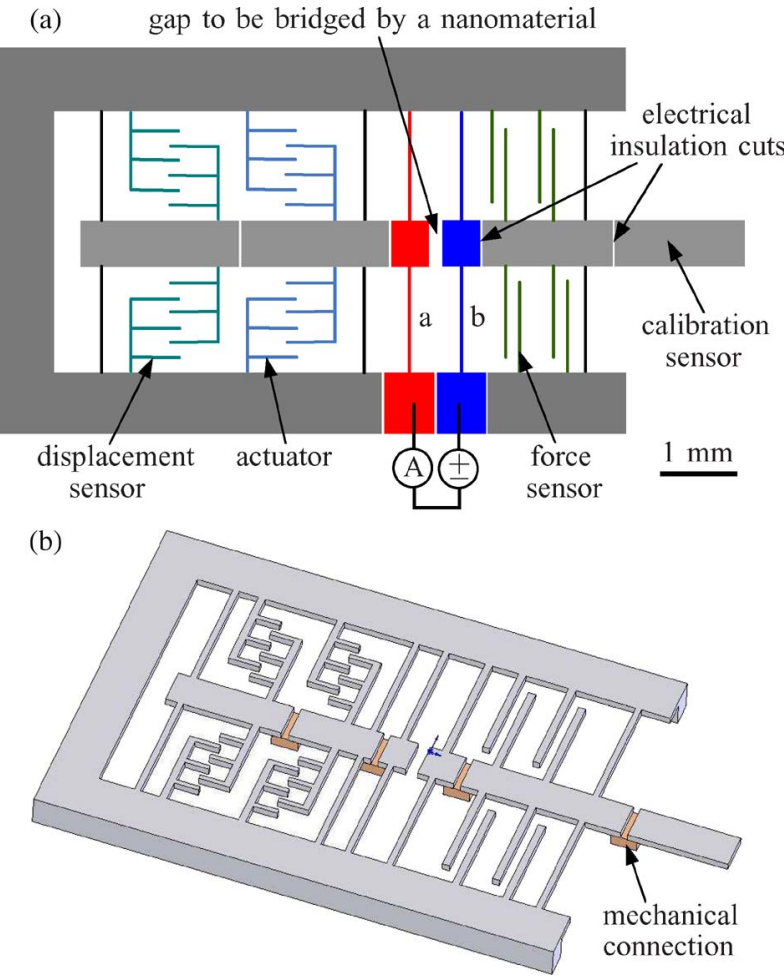

Fig. 1. Schematics of the MEMS device for electromechanical characterization. Force and displacement data of the nanomaterial are both acquired electronically, obviating the reliance on electron microscopy imaging. Electrical insulation within suspended structures enables simultaneous electrical characterization during tensile testing. (a) Planar schematic showing device compositions. (b) Three-dimensional model of the device.

it easier to study the effect of environmental factors, such as gas, light, or temperature, on nanomaterial properties.

The probe (Fig. 1) protruding out from the device frame is for the stiffness calibration of the force sensor. Electrostatic actuator was chosen over electrothermal actuator to avoid unwanted temperature increase of the specimen owing to heat conduction, which can alter material properties. The lateral-comb configuration was used for displacement sensing, since the displacement sensor should have a larger linear displacement sensing range than the force sensor. Additionally, there is an open window below the suspended structures in the area surrounding the gap in order to achieve electron transparency, so that transmission electron microscopy can be used to observe the nanomaterial specimen, if desired.

Fig. 1(a) and (b) also shows four electrical insulation cuts on the actuator shuttle and the force sensor shuttle. These cuts electrically separate structures of different electrical functions. Meanwhile, mechanical connection is maintained by using the insulation layer material below the device silicon layer of the silicon-on-insulator wafer, as shown in Fig. 1(b). The specific functions of these insulation cuts are as follows. First, the insulation cut between the displacement sensor and the actuator ensures that capacitance sensing is decoupled from the actuation voltage. Second, the two electrodes at the gap are, respectively, insulated from the actuator and the force sensor, in order to provide independent electrical connections to the specimen for two-point electrical measurement. During the electrical characterization, an electric current flows in series 


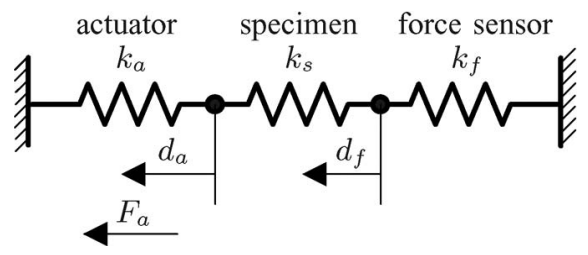

Fig. 2. Mechanical model of the device during mechanical characterization of a specimen. The characteristics (motion range and stiffness) of the actuator and the two sensors must be compatible with that of the target specimen, in order to achieve desired testing conditions.

through beam "b," the specimen, and beam "a" [Fig. 1(a)]. Third, the calibration probe is insulated from the force sensor for valid calibration.

\section{B. Mechanical Analysis}

Since a nanomaterial specimen becomes part of the mechanical system of the device during testing, the motion ranges and stiffness values of the actuator and sensors must be compatible with the mechanical properties of the specimen. Fig. 2 shows a spring diagram for analyzing deformation compatibility and force equilibrium

$$
\begin{aligned}
d_{a} & =d_{s}+d_{f} \\
k_{s} d_{s} & =k_{f} d_{f} \\
F_{a} & =k_{a} d_{a}+k_{s} d_{s}
\end{aligned}
$$

where $d_{a}$ is the displacement of the actuator shuttle; $d_{s}$ is the elongation of the specimen; $d_{f}$ is the displacement of the force sensor shuttle; $k_{a}, k_{s}$, and $k_{f}$ are the stiffness values of the actuator shuttle, the specimen, and the force sensor shuttle, respectively; and $F_{a}$ is the output force generated by the actuator.

The equations were used for parameter design of the device, in consideration of the requirement to produce tensile failure of the specimen. The specimen elongation at the failure point can be estimated using

$$
d_{s o}=w_{g} \varepsilon_{o}
$$

where $w_{g}$ is the gap width of the device (original specimen length) and $\varepsilon_{o}$ is the approximate failure strain according to the reported data in the literature. $d_{f}$ at specimen failure is set to be the largest displacement of the force sensor in its linear sensing range. Then, $k_{s}$ and $k_{f}$ can be selected according to (1b). A slightly larger $k_{f}$ can be chosen to ensure specimen failure. Subsequently, (1a) is used to select $d_{a}$ at specimen failure, which is the largest displacement required for the actuator shuttle. Finally, (1c) is used to determine the largest $F_{a}$ required, for the actuator parameter design (e.g., number of comb pairs).

\section{Electrical Analysis}

Within the circuit for electrical characterization, the specimen is connected in series with beam a and beam $b$ [these beams are labeled in Fig. 1(a)]. Additionally, contact resistances exist between the specimen and the two electrodes (Fig. 3).

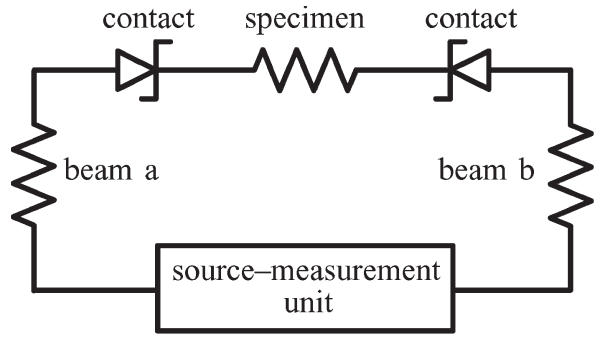

Fig. 3. Circuit diagram of the testing setup during electrical characterization of a nanomaterial specimen. In order to extract the intrinsic electrical characteristics of a specimen, contact resistances as well as the resistances of the two beams are considered.

Thus, the measured current-voltage $(I-V)$ characteristics are not from the specimen alone. The contact can be either an ohmic contact or a Schottky barrier. Since an ohmic contact can also be regarded as a Schottky barrier with a low barrier height close to zero [25], Schottky diodes are used in Fig. 3 to represent contacts. It can be seen that, at a given voltage, one Schottky barrier is forward biased, whereas the other is reverse biased, playing an important role in the measured $I-V$ data. This contact effect must be considered and is discussed in detail in Section IV-C.

The resistances of beam a and beam b can be well calculated, given that beam dimensions and the resistivity of the silicon device layer are known. They can also be experimentally verified by fabricating and testing a device with the actuator shuttle and the force sensor shuttle connected. Additionally, if the device layer silicon is not heavily doped, the piezoresistive effect of beam $a$ and beam $b$ should also be taken into account.

It should be noted that four-point probe measurement is capable of eliminating/minimizing the contact resistance effect [26]-[28] and can also be realized herein by modifying the device design. However, such modification would require a longer specimen to span four suspended electrodes.

\section{Device Fabrication AND CALIBRAtion}

\section{A. Microfabrication}

A deep reactive-ion etching (DRIE) on SOI process, modified from [29], was used for device construction (Fig. 4). The starting SOI wafer has a $25-\mu$ m-thick silicon device layer that was heavily doped with boron (resistivity: $1.7-1.9 \times 10^{-5} \Omega$. $\mathrm{m})$. The low-resistivity device layer reduces the resistivity of beam a and beam b [Figs. 1(a) and 3] as well as their piezoresistive effect.

Briefly, thermal oxidation is used to grow $1-\mu \mathrm{m}$-thick oxide layers on the top and bottom surfaces of the wafer, and then, the top oxide layer is stripped with buffered oxide etch (BOE) [Fig. 4(a)]. The bottom thermal oxide layer is then patterned using reactive-ion etching (RIE) [Fig. 4(b)]. A thick layer of photoresist is patterned on the bottom side, which is used in conjunction with the thermal oxide layer as an etch mask for the DRIE of the handle layer to create steps of a certain height (e.g., $100 \mu \mathrm{m}$ ) [Fig. 4(c)]. The thermal oxide not covered by the photoresist is etched using RIE [Fig. 4(d)]. Using the remained photoresist as an etch mask, the silicon handle layer is etched until the buried oxide layer by DRIE [Fig. 4(e)]. The buried 
(a)

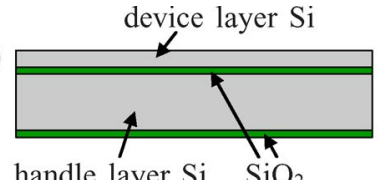

(c)

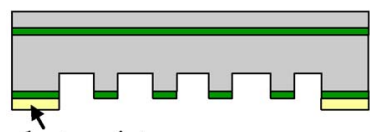

photoresist

(e)

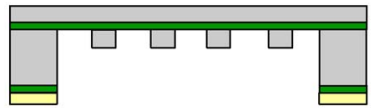

(g)

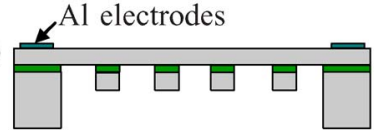

(b)

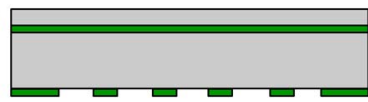

(d)

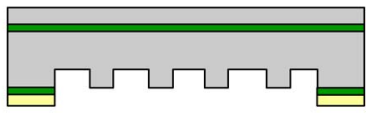

(f)

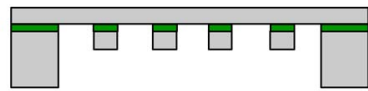

(h)

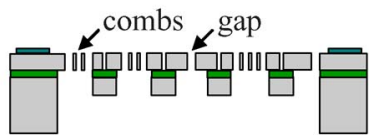

Fig. 4. Microfabrication process flow. (a) Grow thermal oxide on the backside of the SOI wafer. (b) Pattern the thermal oxide layer by RIE. (c) DRIE-etch the handle layer Si. (d) RIE-etch the thermal oxide layer. (e) DRIE-etch the handle layer Si. (f) BOE-etch the buried oxide layer and thermal oxide layer. (g) E-beam-evaporate the Al and lift off. (h) DRIE-etch the device layer Si and release devices.

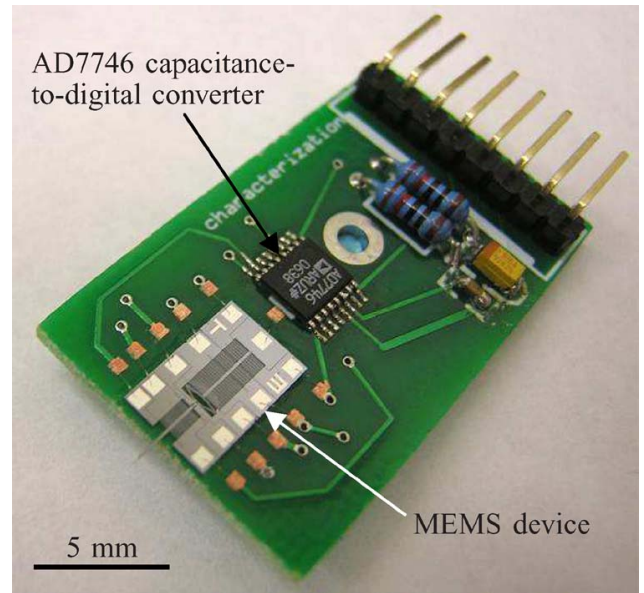

Fig. 5. MEMS device wire bonded to a circuit board with a capacitive readout IC for sampling capacitance data from the displacement sensor and the force sensor.

oxide layer as well as the remained bottom oxide layer is etched using BOE [Fig. 4(f)]. Aluminum electrodes are patterned on the top side by e-beam evaporation and lift off [Fig. 4(g)]. Finally, the device layer is patterned using DRIE to form active structures [Fig. 4(h)].

For mechanically connecting suspended structures with electrical insulation, the two-step DRIE process of the handle layer [Fig. 4(c) and (e)] creates a step difference between the central suspended structure and the device frame, which significantly reduces the risk of device breakage during device handling and operation. The released devices are glued and wire bonded to custom-made printed circuit boards (Fig. 5) with a two-channel capacitive-to-digital converter (AD7746, Analog Devices Inc.) for sampling data from the two capacitive sensors on the device. Since the remained flux on the circuit boards can outgas in SEM, it was removed using an ultrasonic cleaner and an aqueous flux remover, prior to the wire bonding. The glue and solder used in this work are vacuum compatible.

A few devices whose actuator shuttle and force sensor shuttle are connected by design were also included on the same wafer, for device characterization. The equivalent resistance of beam $\mathrm{a}$ and beam $\mathrm{b}$ was measured to be $81 \Omega$, which is negligible in comparison with the nanowire resistances obtained in Section IV-C. Furthermore, the deformation of the two beams by the actuator did not result in resistance change. Therefore, beam a and beam $b$ can be safely ignored in the circuit shown in Fig. 3.

\section{B. Sensor Calibration}

The displacement sensing functions of both the displacement sensor and the force sensor were calibrated. The force sensing function of the force sensor was also calibrated. A data sampling rate of $45 \mathrm{~Hz}$ was used during device calibration.

The displacement sensor was deformed by driving the actuator [Fig. 1(a)], with the displacements measured from microscopy imaging and correlated to the output voltage of the readout circuit [Fig. 6(a)]. Determined from the noise level of the output voltage, the displacement sensor exhibits a resolution of $1.7 \mathrm{~nm}$ at $45 \mathrm{~Hz}$. The force sensor was deformed by using a microprobe under a probe station to push the calibration probe of the device, with the displacements also measured from both imaging and the readout circuit. The calibration results are shown in Fig. 6(b). The displacement sensing resolution of the force sensor was determined to be $1.5 \mathrm{~nm}$ at $45 \mathrm{~Hz}$.

A precision microbalance (XS105DU, Mettler-Toledo Inc.) with a resolution of $0.1 \mu \mathrm{N}$ was used to calibrate the force sensor. Fig. 6(c) shows the calibration results. The force sensor exhibits a resolution of $26.8 \mathrm{nN}$ at $45 \mathrm{~Hz}$. Additionally, the stiffness of the force sensor can be obtained by calculating the ratio of the slope of the regression line in Fig. 6(b) to that in Fig. 6(c), resulting in $18.2 \mathrm{~N} / \mathrm{m}$.

\section{EXPERIMENTAL RESULTS AND DISCUSSIONS}

\section{A. Synthesis of Silicon Nanowires}

As a type of piezoresistive nanomaterial, silicon nanowires were chosen for characterization by the MEMS device in this paper. Silicon nanowires used in this paper were vaporliquid-solid (VLS) synthesized using low-pressure chemical vapor deposition. A gold thin film was thermally evaporated onto a silicon (111) substrate, which was subsequently annealed to form a discontinuous film consisting of 50-100-nm-diameter gold islands. The substrate was then introduced into a reactor and brought to a temperature above the $\mathrm{Au}-\mathrm{Si}$ eutectic point of $363{ }^{\circ} \mathrm{C}$ in a $\mathrm{H}_{2}$ atmosphere. The deposition occurred at approximately $550{ }^{\circ} \mathrm{C}$ using a $10 \% \mathrm{SiH}_{4} / \mathrm{H}_{2}$ as the silicon source with trace levels of phosphine $\left(\mathrm{PH}_{3}\right)$ as the n-type dopant source at a total pressure between 10 and 50 torr. Lengths of the synthesized nanowires were 10-30 $\mu \mathrm{m}$.

\section{B. Transfer of Nanowire Onto MEMS Device}

A few methods have been reported for the integration of a nanomaterial to a MEMS device. Nanomaterials can be fabricated directly on MEMS devices [9], [30]. Presynthesized 

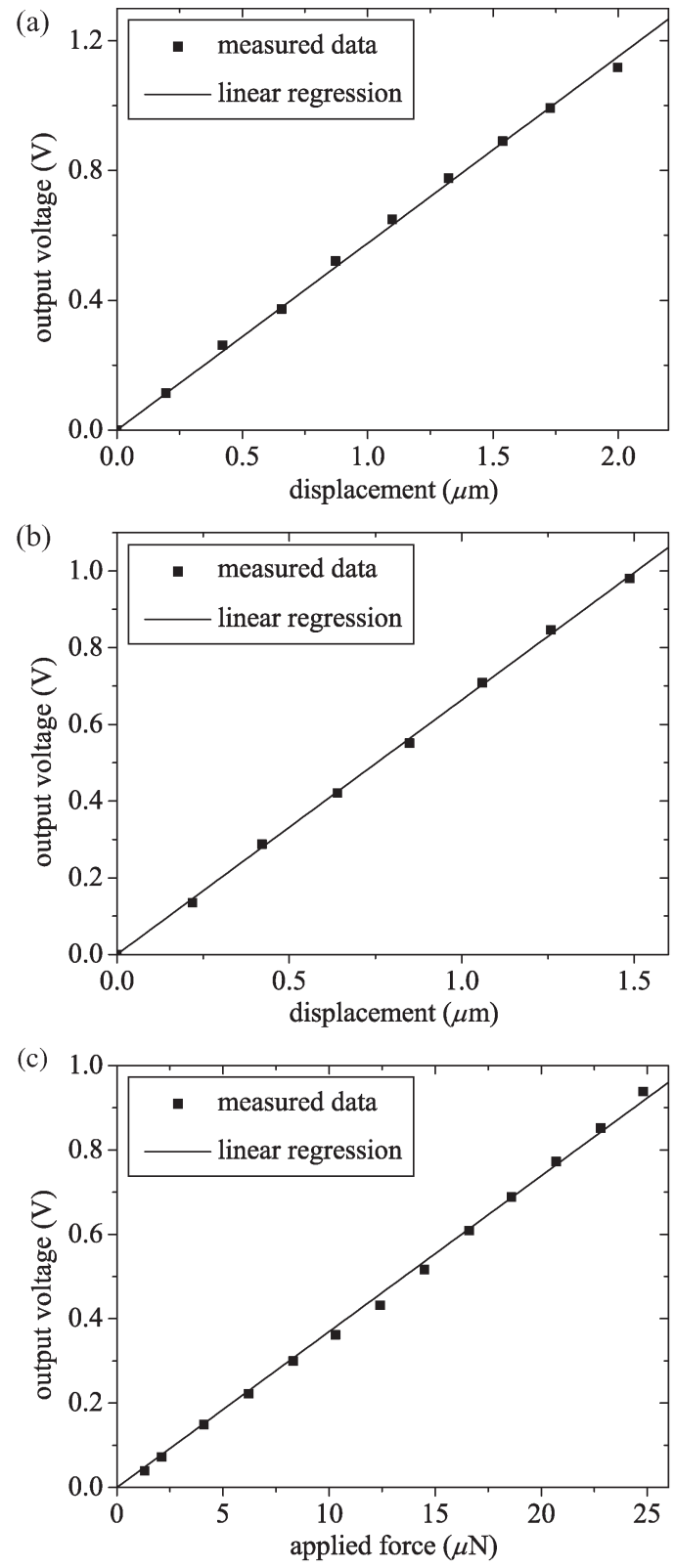

Fig. 6. Calibration results of (a) the displacement sensor and (b) and (c) the force sensor. The displacement sensor exhibits a resolution of $1.7 \mathrm{~nm}$ at $45 \mathrm{~Hz}$. The force sensor exhibits a displacement sensing resolution of $1.5 \mathrm{~nm}$ and a force sensing resolution of $26.8 \mathrm{nN}$ at $45 \mathrm{~Hz}$.

nanomaterials were also transferred onto MEMS devices via dielectrophoresis trapping [12], [22], [31], focused-ion-beam deposition [14], and direct pick and place [11], [13], [17], [20], [32]-[35]. Due to the flexibility of pick-and-place nanomanipulation inside SEM and no need for nanomaterial preprocessing (e.g., sonication), we transferred individual silicon nanowires from growth substrates onto MEMS devices via direct pick and place.

For the experimental setup, a silicon nanowire substrate and a circuit board with a wire-bonded MEMS device were placed on the specimen stage of a SEM (S-4000, Hitachi, Ltd.), where a nanomanipulation system (S100, Zyvex Instruments) was mounted, as illustrated in Fig. 7(a). A tungsten probe (tip diameter: $200 \mathrm{~nm}$ ) was installed to one of the nanomanipulators
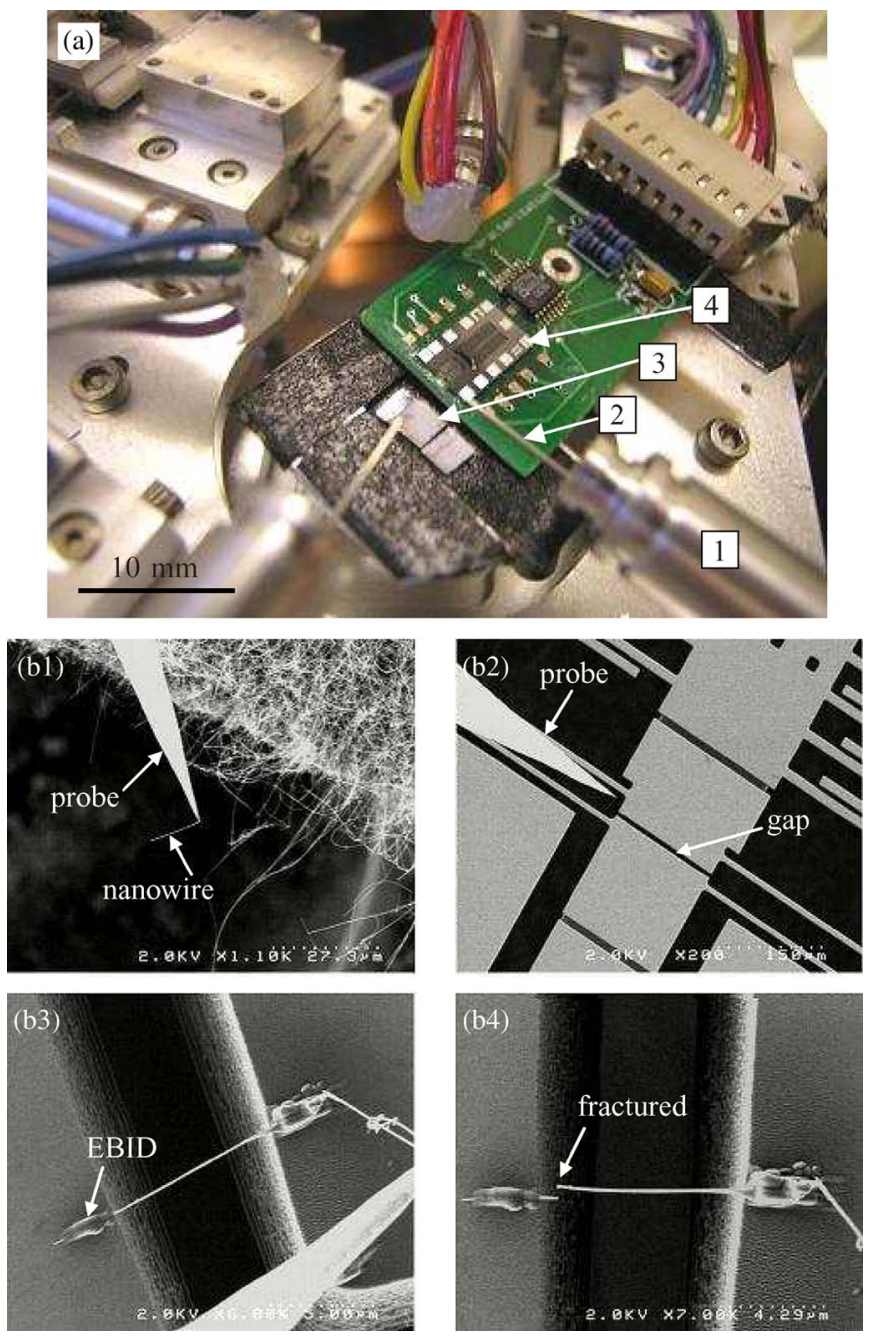

Fig. 7. Nanowire transfer. (a) Experimental setup: The MEMS device and the nanowire sample are installed to the specimen stage of a SEM integrated with a nanomanipulation system. (1) Nanomanipulator, (2) tungsten nanoprobe, (3) silicon nanowire substrate, and (4) MEMS device. (b) Transfer procedure: A silicon nanowire is transferred from its growth substrate to the testing device via nanomanipulation inside a SEM, followed by electromechanical characterization. (b1) Probe picks up a nanowire by soldering it to the probe tip using EBID and detaching it from the growth substrate. (b2) Nanomanipulator transfers the nanowire to above the device. (b3) Nanomanipulator places the nanowire across the gap of the device, and the nanowire is EBID soldered to the two edges of the gap. (b4) Device electromechanically interrogates the nanowire until its tensile failure.

and used to transfer individual nanowires [Fig. 7(b1)-(b3)]. Throughout the manipulation process, an acceleration voltage of $2 \mathrm{kV}$ in SEM imaging was typically used.

Briefly, the nanomanipulator first approaches an edge of the nanowire substrate and establishes contact with a single nanowire near its root. Following the contact, the nanowire is "soldered" to the probe tip using e-beam induced deposition (EBID). The deposited material from EBID without injecting precursors is carbonaceous material, which is from the decomposition of contaminants inside the SEM chamber [36]-[38]. The probe then retracts and pulls the nanowire off from the growth substrate, as shown in Fig. 7(b1). A detached nanowire typically fractures near its root, so the upper section of the nanowire to be used for testing does not experience tension and remains intact. 

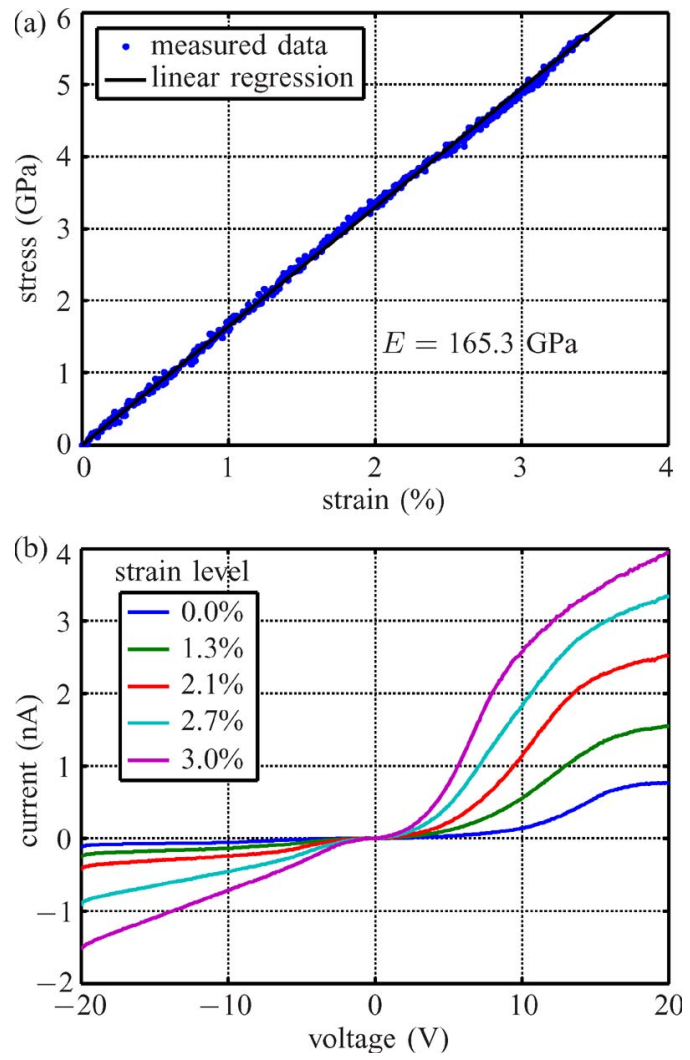

Fig. 8. Mechanical and electrical characterization results. (a) Stress-strain curve. The nanowire exhibits a Young's modulus of $165.3 \mathrm{GPa}$ and a failure strength of $5.67 \mathrm{GPa}$. (b) $I-V$ characteristics of the nanowire under different strain levels.

The nanomanipulator subsequently transfers the nanowire to above the MEMS device [Fig. 7(b2)] and lowers the nanowire to place it across the gap of the device. The first bond between the nanowire and one edge of the gap is formed again via EBID. The nanomanipulator then orients the nanowire to make it perpendicular to the gap, followed by EBID soldering the nanowire to the second gap edge [Fig. 7(b3)]. The purpose of fixing the nanowire to the very edges of the gap, rather than some distance away from the edges, is to prevent static friction between the nanowire and the device surface when the nanowire is stretched.

Finally, the probe is retracted from the MEMS device, during which the nanowire breaks between the second EBID bond on the device and the EBID bond on the probe. Prior to stretching the nanowire, the two bonds are strengthened using EBID once again, until the $I-V$ characteristics of the nanowire do not change anymore. This step was also performed to ensure that the EBID bonds are secure during nanowire stretching and do not break before the tensile failure of the nanowire.

\section{Nanowire Characterization}

The nanowire specimen was tensile stretched until its fracture [Fig. 7(b4)], with the force-elongation data recorded, during which its $I-V$ data curves were also obtained using a source measurement unit (SourceMeter 2602, Keithley Instruments Inc.) at a number of strain levels. A representative stress-strain curve from a nanowire specimen is shown in Fig. 8(a), from which the Young's modulus (165.3 GPa) and failure strength (5.67 GPa) were determined. It can also be observed from the curve that the nanowire specimen did not experience the phase of plastic deformation before fracture, proving to be brittle.

The Young's modulus derived from five silicon nanowires was $165.4 \pm 3.9 \mathrm{GPa}(n=5)$, which is in agreement with the findings for VLS-grown [111] silicon nanowires as reported in [20] and [39]. In all experiments, the nanowire misalignment was less than $5^{\circ}$ between the axial direction of the nanowire specimen and the stretching direction, resulting in an error of less than $1 \%$ in the measured Young's modulus [11]. The failure strengths of the nanowires were determined to be $5.3 \pm 0.6 \mathrm{GPa}$ $(n=5)$. The tested nanowires had diameters of $72-97 \mathrm{~nm}$ and lengths of $6.7-8.2 \mu \mathrm{m}$ between the two EBID bonds.

For coupled electrical characterization at each strain level, a voltage sweep (e.g., from -20 to $+20 \mathrm{~V}$ ) was applied to a nanowire specimen, and the current flow was measured. Fig. 8(b) shows the $I-V$ characteristics of a silicon nanowire at different strain levels. It can be seen that straining the nanowire resulted in $I-V$ changes.

Fig. 8(b) also shows that the $I-V$ curves are not symmetrical with regard to the origin, indicating the existence of Schottky contacts. When the applied voltage is low $(<0.5 \mathrm{~V})$, the voltage is mainly distributed on the two Schottky barriers (Fig. 3), rather than on the specimen. As the applied voltage increases, the specimen starts to contribute more to the $I-V$ characteristics. At a high voltage $(>5 \mathrm{~V})$, while the voltage drop across the forward-biased Schottky barrier remains small, the voltage drop across the reverse-biased Schottky barrier becomes saturated. Thus, the slope of the linear section of an $I-V$ curve at high voltages approximates the conductance of the specimen [25], [40]-[42]. When the applied voltage is even higher (e.g., $>15 \mathrm{~V}$ ), the $I-V$ curves become more nonlinear since the electrical transport through the nanowire is space-charge limited [6], [43].

Therefore, the voltage range of $5-9 \mathrm{~V}$ in Fig. 8(b) was used to determine the intrinsic resistance of the nanowire at different strain levels. The resistance and resistivity under the unstrained condition were determined to be $5.9 \times 10^{11} \Omega$ and $406 \Omega \cdot \mathrm{m}$. At $3.0 \%$ strain, the resistance was determined to be $2.2 \times 10^{10} \Omega$, reduced by a factor of 26.8 from the resistance at zero strain. This significant change in the resistance indicates the piezoresistive effect of the silicon nanowire. As another measure to quantify this effect, the gauge factor (ratio of relative resistance change to strain) of the nanowire was determined to be 67.1 at $1.3 \%$ strain. The characterization of the piezoresistivity of silicon nanowire was enabled by the capability of the MEMS device for simultaneous electrical and mechanical characterization.

\section{Effect of E-Beam Irradiation}

Repeated tensile tests were performed on silicon nanowires, under the conditions of e-beam on and off. The experimental results indicate that e-beam irradiation did not affect the measured mechanical properties. However, e-beam irradiation produces significant effect on the $I-V$ characteristics of nanowires. In our study, $I-V$ characteristics of the nanowires were quantified 

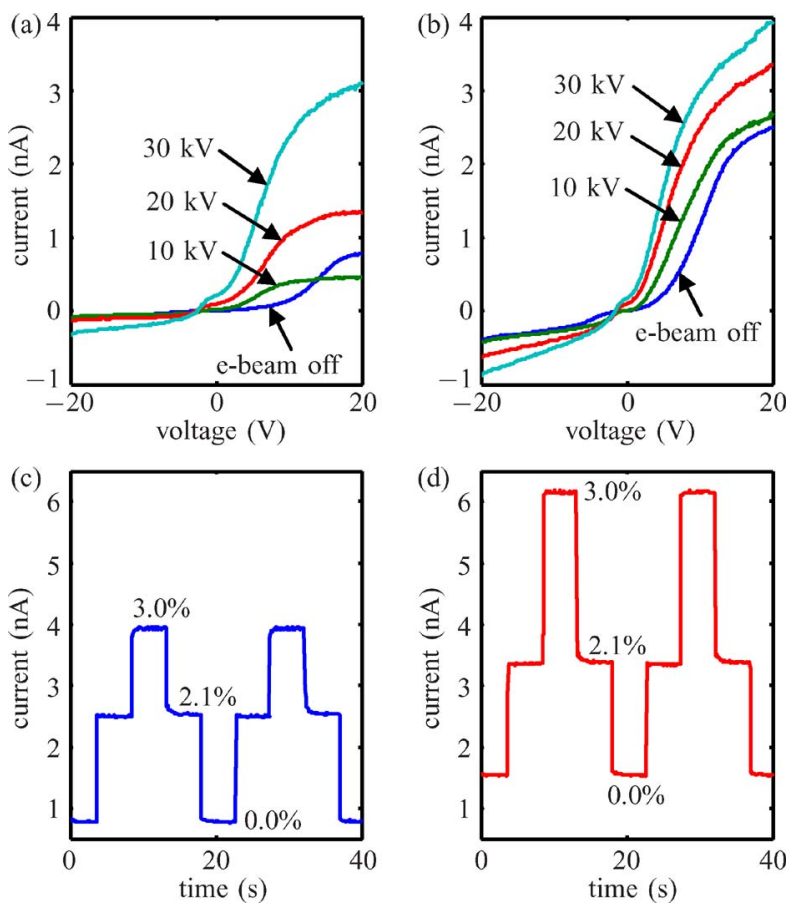

Fig. 9. (a and b) $I-V$ characteristics of a nanowire under e-beam irradiation of different acceleration voltages $(0,10,20$, and $30 \mathrm{kV})$, with nanowire (a) unstrained and (b) $2.1 \%$ strain, revealing the effect of SEM imaging. (c and d) Dynamic current response of a nanowire subject to cyclic mechanical loading with different strain levels $(0 \%, 2.1 \%$, and $3.0 \%)$, under a constant applied voltage of $20 \mathrm{~V}$, with (c) e-beam off and (d) e-beam on with the acceleration voltage of $20 \mathrm{kV}$.

with the e-beam off and on with different acceleration voltages of 10, 20, and $30 \mathrm{kV}$, under $0 \%$ and $2.1 \%$ strain levels [Fig. 9(a) and (b)]. It can be seen that e-beam irradiation significantly alters $I-V$ data, likely through charge injection from e-beam into the specimen. Thus, for electromechanical characterization of nanowires, the elongation measurement preferably should be performed electronically. When EM imaging must be used, the acceleration voltage should be kept low.

Fig. 9(c) and (d) shows the data collected on a silicon nanowire that was subject to cyclic loading at strain levels of $0 \%, 2.1 \%$, and $3.0 \%$. By comparing the e-beam off result [Fig. 9(c)] with the e-beam on result [Fig. 9(d)], the current increases due to e-beam irradiation are $107 \%$ for $0 \%$ strain, $35 \%$ for $2.1 \%$ strain, and $56 \%$ for $3.0 \%$ strain, again demonstrating the significant effect of e-beam irradiation. Additionally, cyclic loading was repeated for over 1000 cycles. No signs of nanowire fatigue were observed.

Of the over 1000 cycles, the aforementioned e-beam on and e-beam off experiments [Fig. 9(c) and (d)] demonstrated highly repeatable results, indicating that short-term e-beam irradiation affects electrical measurements but does not permanently change the electrical properties of the nanowires.

\section{CONCLUSION}

This paper has described a MEMS device for simultaneous electrical and mechanical characterization of individual nanowires. The device integrates an actuator, two capacitive sensors, and two suspended electrodes for a nanowire to bridge.
Tensile forces and elongation measurements are all acquired electronically, without relying on EM imaging. The two suspended electrodes enable $I-V$ characteristics of a specimen to be obtained at different strain levels. Nanomanipulation (pick and place) inside SEM was performed to transfer individual silicon nanowires from their growth substrates onto the MEMS device. Measurements were made to quantify the mechanical and electrical properties and the piezoresistive effect of the silicon nanowires. The significant effect that e-beam irradiation has through EM imaging on the $I-V$ characteristics of nanowires has been also revealed.

\section{REFERENCES}

[1] C. Stampfer, T. Helbling, D. Obergfell, B. Schöberle, M. K. Tripp, A. Jungen, S. Roth, V. M. Bright, and C. Hierold, "Fabrication of singlewalled carbon-nanotube-based pressure sensors," Nano Lett., vol. 6, no. 2, pp. 233-237, Feb. 2006.

[2] C. Stampfer, A. Jungen, R. Linderman, D. Obergfell, S. Roth, and C. Hierold, "Nano-electromechanical displacement sensing based on single-walled carbon nanotubes," Nano Lett., vol. 6, no. 7, pp. 1449-1453, Jul. 2006.

[3] T. W. Tombler, C. Zhou, L. Alexseyev, J. Kong, H. Dai, L. Liu, C. S. Jayanthi, M. Tang, and S.-Y. Wu, "Reversible electromechanical characteristics of carbon nanotubes under local-probe manipulation," Nature, vol. 405, no. 6788, pp. 769-772, Jun. 2000.

[4] J. Cao, Q. Wang, and H. Dai, "Electromechanical properties of metallic, quasimetallic, and semiconducting carbon nanotubes under stretching," Phys. Rev. Lett., vol. 90, no. 15, pp. 157 601-1-157 601-4, Apr. 2003.

[5] R. He and P. Yang, "Giant piezoresistance effect in silicon nanowires," Nat. Nanotechnol., vol. 1, no. 1, pp. 42-46, Oct. 2006.

[6] A. Lugstein, M. Steinmair, A. Steiger, H. Kosina, and E. Bertagnolli, "Anomalous piezoresistance effect in ultrastrained silicon nanowires," Nano Lett., vol. 10, no. 8, pp. 3204-3208, Aug. 2010.

[7] P. Neuzil, C. C. Wong, and J. Reboud, "Electrically controlled giant piezoresistance in silicon nanowires," Nano Lett., vol. 10, no. 4, pp. 1248 1252, Apr. 2010.

[8] G. Hwang, H. Hashimoto, D. J. Bell, L. Dong, B. J. Nelson, and S. Schön, "Piezoresistive InGaAs/GaAs nanosprings with metal connectors," Nano Lett., vol. 9, no. 2, pp. 554-561, Feb. 2009.

[9] M. A. Haque and M. T. A. Saif, "Microscale materials testing using MEMS actuators," J. Microelectromech. Syst., vol. 10, no. 1, pp. 146-152, Mar. 2001.

[10] P. A. Williams, S. J. Papadakis, M. R. Falvo, A. M. Patel, M. Sinclair, A. Seeger, A. Helser, and R. M. Taylor, II, "Controlled placement of an individual carbon nanotube onto a microelectromechanical structure," Appl. Phys. Lett., vol. 80, no. 14, pp. 2574-2576, Apr. 2002.

[11] Y. Zhu and H. D. Espinosa, "An electromechanical material testing system for in situ electron microscopy and applications," Proc. Nat. Acad. Sci., vol. 102, no. 41, pp. 14 503-14 508, Oct. 2005.

[12] S. Lu, Z. Guo, W. Ding, D. A. Dikin, J. Lee, and R. S. Ruoff, "In situ mechanical testing of templated carbon nanotubes," Rev. Sci. Instrum. vol. 77, no. 12, pp. $125101-1-125$ 101-6, Dec. 2006.

[13] H. D. Espinosa, Y. Zhu, and N. Moldovan, "Design and operation of a MEMS-based material testing system for nanomechanical characterization," J. Microelectromech. Syst., vol. 16, no. 5, pp. 1219-1231, Oct. 2007.

[14] M. Kiuchi, S. Matsui, and Y. Isono, "Mechanical characteristics of FIB deposited carbon nanowires using electrostatic actuated nanotensile testing device," J. Microelectromech. Syst., vol. 16, no. 2, pp. 191-201, Apr. 2007.

[15] B. Peng, M. Locascio, P. Zapol, S. Li, S. L. Mielke, G. C. Schatz, and H. D. Espinosa, "Measurements of near-ultimate strength for multiwalled carbon nanotubes and irradiation-induced crosslinking improvements," Nat. Nanotechnol., vol. 3, pp. 626-631, Oct. 2008.

[16] M. Kiuchi, S. Matsui, and Y. Isono, "The piezoresistance effect of FIB-deposited carbon nanowires under severe strain," J. Micromech. Microeng., vol. 18, no. 6, p. 065 011, Jun. 2008.

[17] J. J. Brown, J. W. Suk, G. Singh, A. I. Baca, D. A. Dikin, R. S. Ruoff, and V. M. Bright, "Microsystem for nanofiber electromechanical measurements," Sens. Actuators A, Phys., vol. 155, no. 1, pp. 1-7, Oct. 2009.

[18] R. Agrawal, B. Peng, and H. D. Espinosa, "Experimental-computational investigation of $\mathrm{ZnO}$ nanowires strength and fracture," Nano Lett., vol. 9, no. 12 , pp. $4177-4183$, Dec. 2009. 
[19] D. Zhang, J.-M. Breguet, R. Clavel, L. Phillippe, I. Utke, and J. Michler, "In situ tensile testing of individual Co nanowires inside a scanning electron microscope," Nanotechnology, vol. 20, no. 36, p. 365706 , Sep. 2009.

[20] D. Zhang, J.-M. Breguet, R. Clavel, V. Sivakov, S. Christiansen, and J. Michler, "In situ electron microscopy mechanical testing of silicon nanowires using electrostatically actuated tensile stages," J. Microelectromech. Syst., vol. 19, no. 3, pp. 663-674, Jun. 2010.

[21] M. A. Haque, H. D. Espinosa, and H. J. Lee, "MEMS for in situ testing-Handling, actuation, loading, and displacement measurements," MRS Bull., vol. 35, pp. 375-381, May 2010.

[22] J. Brown, A. Baca, K. Bertness, D. Dikin, R. Ruoff, and V. Bright, "Tensile measurement of single crystal gallium nitride nanowires on MEMS test stages," Sens. Actuators A, Phys., vol. 166, no. 2, pp. 177-186, Apr. 2011. DOI:10.1016/j.sna.2010.04.002.

[23] W. Kang, J. H. Han, and M. T. A. Saif, "A novel method for in situ uniaxial tests at the micro/nano scale-Part II: Experiment," J. Microelectromech. Syst., vol. 19, no. 6, pp. 1322-1330, Dec. 2010.

[24] R. A. Bernal, R. Agrawal, B. Peng, K. A. Bertness, N. A. Sanford, A. V. Davydov, and H. D. Espinosa, "Effect of growth orientation and diameter on the elasticity of GaN nanowires. A combined in situ TEM and atomistic modeling investigation," Nano Lett., vol. 11, no. 2, pp. 548-555, Feb. 2011. dx.doi.org/10.1021/n1103450e.

[25] Z. Zhang, K. Yao, Y. Liu, C. Jin, X. Liang, Q. Chen, and L.-M. Peng, "Quantitative analysis of current-voltage characteristics of semiconducting nanowires: Decoupling of contact effects," Adv. Funct. Mater., vol. 17, no. 14, pp. 2478-2489, Sep. 2007.

[26] S. Yoshimoto, Y. Murata, K. Kubo, K. Tomita, K. Motoyoshi, T. Kimura, H. Okino, R. Hobara, I. Matsuda, S. Honda, M. Katayama, and S. Hasegawa, "Four-point probe resistance measurements using PtIr-coated carbon nanotube tips," Nano Lett., vol. 7, no. 4, pp. 956-959, Apr. 2007.

[27] C. Ru, Y. Zhang, Y. Sun, Y. Zhong, X. Sun, D. Hoyle, and I. Cotton, "Automated four-point probe measurement of nanowires inside a scanning electron microscope," IEEE Trans. Nanotechnol., vol. 10, no. 4, pp. 674681, Jul. 2011

[28] T.-H. Kim, X.-G. Zhang, D. M. Nicholson, B. M. Evans, N. S. Kulkarni, B. Radhakrishnan, E. A. Kenik, and A.-P. Li, "Large discrete resistance jump at grain boundary in copper nanowire," Nano Lett., vol. 10, no. 8, pp. 3096-3100, Aug. 2010.

[29] Y. Zhang, B. K. Chen, X. Liu, and Y. Sun, "Autonomous robotic pick-andplace of microobjects," IEEE Trans. Robot., vol. 26, no. 1, pp. 200-207, Feb. 2010.

[30] M. A. Haque and M. T. A. Saif, "Deformation mechanisms in freestanding nanoscale thin films: A quantitative in situ transmission electron microscope study," Proc. Nat. Acad. Sci., vol. 101, no. 17, pp. 6335-6340, Apr. 2004.

[31] S. Lu, J. Chung, and R. S. Ruoff, "Controlled deposition of nanotubes on opposing electrodes," Nanotechnology, vol. 16, no. 9, pp. 1765-1770, Sep. 2005.

[32] B. D. Sosnowchik, J. Chang, and L. Lin, "Pick, break, and placement of one-dimensional nanostructures for direct assembly and integration," Appl. Phys. Lett., vol. 96, no. 15, pp. 153 101-1-153 101-3, Apr. 2010.

[33] Y. Ganesan, Y. Lu, C. Peng, H. Lu, R. Ballarini, and J. Lou, "Development and application of a novel microfabricated device for the in situ tensile testing of 1-D nanomaterials," J. Microelectromech. Syst., vol. 19, no. 3, pp. 675-682, Jun. 2010.

[34] Y. Ganesan, C. Peng, Y. Lu, L. Ci, A. Srivastava, P. M. Ajayan, and J. Lou, "Effect of nitrogen doping on the mechanical properties of carbon nanotubes," ACS Nano, vol. 4, no. 12, pp. 7637-7643, Dec. 2010.

[35] Y. Hu, J. Zhou, P.-H. Yeh, Z. Li, T.-Y. Wei, and Z. L. Wang, "Supersensitive, fast-response nanowire sensors by using Schottky contacts," Adv. Mater., vol. 22, no. 30, pp. 3327-3332, Aug. 2010.

[36] M.-F. Yu, O. Lourie, O. Lourie, K. Moloni, T. F. Kelly, and R. S. Ruoff, "Strength and breaking mechanism of multiwalled carbon nanotubes under tensile load," Science, vol. 287, no. 5453, pp. 637-640, Jan. 2000.

[37] T. Fukuda, F. Arai, and L. Dong, "Assembly of nanodevices with carbon nanotubes through nanorobotic manipulations," Proc. IEEE, vol. 91, no. 11, pp. 1803-1818, Nov. 2003.

[38] T. Fukuda, M. Nakajima, P. Liu, and H. ElShimy, "Nanofabrication, nanoinstrumentation and nanoassembly by nanorobotic manipulation," Int. J. Robot. Res., vol. 28, no. 4, pp. 537-547, Apr. 2009.

[39] M. J. Gordon, T. Baron, F. Dhalluin, P. Gentile, and P. Ferret, "Size effects in mechanical deformation and fracture of cantilevered silicon nanowires," Nano Lett., vol. 9, no. 2, pp. 525-529, Feb. 2009.
[40] Z. Y. Zhang, C. H. Jin, X. L. Liang, Q. Chen, and L.-M. Peng, "Current-voltage characteristics and parameter retrieval of semiconducting nanowires," Appl. Phys. Lett, vol. 88, no. 7, pp. 073 102-1-073 102-3, Feb. 2006.

[41] Y.-F. Lin and W.-B. Jian, "The impact of nanocontact on nanowire based nanoelectronics," Nano Lett., vol. 8, no. 10, pp. 3146-3150, Oct. 2008.

[42] A. Asthana, T. Shokuhfar, Q. Gao, P. Heiden, C. Friedrich, and R. S. Yassar, "A study on the modulation of the electrical transport by mechanical straining of individual titanium dioxide nanotube," Appl. Phys. Lett, vol. 97, no. 7, pp. 072 107-1-072 107-3, Aug. 2010.

[43] A. A. Grinberg, S. Luryi, M. R. Pinto, and N. L. Schryer, "Space-chargelimited current in a film," IEEE Trans. Electron Devices, vol. 36, no. 6, pp. 1162-1170, Jun. 1989.

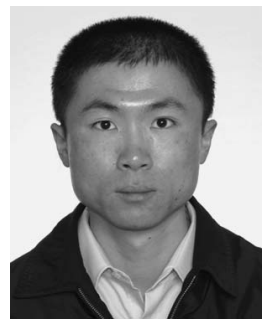

Yong Zhang (S'09) received the B.S. and M.S. degrees in mechatronics engineering from Harbin Institute of Technology, Harbin, China, in 2005 and 2007 , respectively. He is currently working toward the Ph.D. degree in the Department of Electrical and Computer Engineering, University of Toronto, Toronto, ON, Canada.

His research interests include manipulation and characterization of micrometer- and nanometer-sized materials using microelectromechanical systems and robotics.

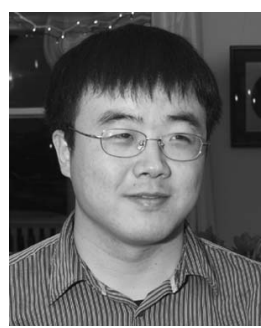

Xinyu Liu (S'06-M'10) received the B.Eng. and M.Eng. degrees in mechanical engineering from Harbin Institute of Technology, Harbin, China, in 2002 and 2004, respectively, and the Ph.D. degree in mechanical engineering from the University of Toronto, Toronto, ON, Canada, in 2009.

He was a Postdoctoral Fellow in the Department of Chemistry and Chemical Biology, Harvard University, Cambridge, MA. He is currently an Assistant Professor in the Department of Mechanical Engineering, McGill University, Montreal, QC, Canada. His research interests are design and fabrication of microelectromechanical systems devices, micro- and nanorobotic manipulation of bio- and nanomaterials, low-cost diagnostic technologies for the developing world, and microfluidics for bioapplications.

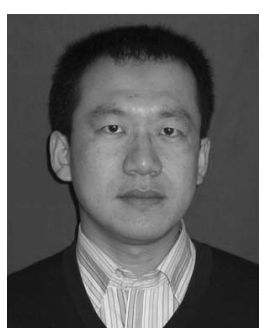

Changhai Ru (M'07) received the Ph.D. degree in mechatronics engineering from Harbin Institute of Technology, Harbin, China, in 2005.

He was a Postdoctoral Fellow in the Department of Mechanical and Industrial Engineering, University of Toronto, Toronto, ON, Canada. He is currently a Professor at the Robotics and Microsystems Center, Soochow University, Suzhou, China. His research interests include micro-/nanomanipulation, nanopositioning technology, and solid-state actuators' driving and control methods.

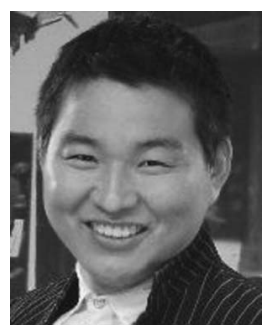

Yan Liang Zhang (M'10) received the B.Eng. degree in computer engineering and the Ph.D. degree in mechanical and aerospace engineering from Nanyang Technological University, Singapore, in 2006 and 2010, respectively.

He is currently a Postdoctoral Fellow in the Department of Mechanical and Industrial Engineering, University of Toronto, Toronto, ON, Canada. His research interests include cell mechanobiology, robotics automation under optical and electron microscopes for micro-/nanomanipulation, and fabrication and characterization of nanodevices. 


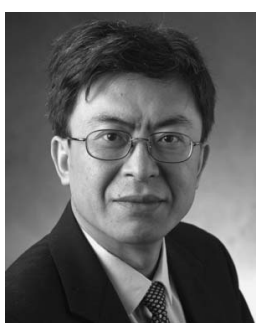

Lixin Dong (S'01-M'03-SM'10) received the B.S. and M.S. degrees in mechanical engineering from Xi'an University of Technology (XUT), Xi'an, China, in 1989 and 1992, respectively, and the Ph.D. degree in microsystems engineering from Nagoya University, Nagoya, Japan, in 2003.

He was with XUT, where he became a Research Associate in 1992, a Lecturer in 1995, and an Associate Professor in 1998. In 2003, he became an Assistant Professor at Nagoya University. In 2004, he joined the Swiss Federal Institute of Technology Zürich (ETH Zürich), Zürich, Switzerland, as a Research Scientist, where he became a Senior Research Scientist in 2005 and led the NanoRobotics Group, Institute of Robotics and Intelligent Systems. Since December 2008, he has been an Assistant Professor at Michigan State University, East Lansing. His current research interests include nanorobotics, nanoelectromechanical systems, mechatronics, mechanochemistry, and nanobiomedical devices.

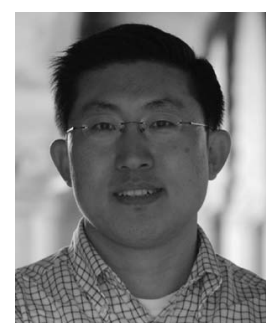

Yu Sun (S'01-M'03-SM'07) received the B.S. degree in electrical engineering from Dalian University of Technology, Dalian, China, in 1996, the M.S. degree from Institute of Automation, Chinese Academy of Sciences, Beijing, China, in 1999, and the M.S. degree in electrical engineering and the Ph.D. degree in mechanical engineering from the University of Minnesota, Minneapolis, in 2001 and 2003, respectively.

$\mathrm{He}$ is currently an Associate Professor in the Department of Mechanical and Industrial Engineering and is jointly appointed in the Institute of Biomaterials and Biomedical Engineering and the Department of Electrical and Computer Engineering, University of Toronto, Toronto, ON, Canada. His research areas include the design and fabrication of micro- and nanoelectromechanical systems devices, micro-/nanorobotic manipulation, and the manipulation and characterization of biological cells, biomolecules, and nanomaterials. He was a Research Scientist at the Swiss Federal Institute of Technology Zürich (ETH Zürich), Zürich, Switzerland, before joining the faculty of the University of Toronto in July 2004. He is the Canada Research Chair in micro- and nanoengineering systems and a Guest Chair Professor at Soochow University, Suzhou, China. 